\title{
Comparison of tactual and visual cues on the visual cliff ${ }^{1}$
}

\author{
H. R. SCHIFFMAN, RUTGERS UNIVERSITY \\ BERNARD BEER, ARTHUR KOENIG, DONALD E. CLODY, SQUIBB INSTITUTE FOR MEDICAL RESEARCH
}

\begin{abstract}
The visual cliff apparatus was used to examine the role of tactual cues in depth discrimination. The height of the centerboard and the presence or absence of the glass surface covering the deep and shallow sides were varied. In general, the results indicate that the adult rat will use tactual information for depth discrimination on the visual cliff and that the degree of its use varies with its availability.
\end{abstract}

A central problem to investigators of depth perception who use the visual cliff apparatus (Walk \& Gibson, 1961 ) is the determination of the kinds of cues or stimuli that are effectively utilized. Although all species tested on the cliff exhibited some depth discrimination, it is likely that different species differentially emphasize the available cues to depth. This follows from the fact that many of the animals tested attain optical proficiency at different stages of maturation, possess different sensory anatomies, and habituate in differently illuminated environments. For example, the diurnal chick shows correlated visual and locomotor ability shortly after hatching while the nocturnal rat requires a period of development in order to perform adequate visual-motor activities. Moreover, the rat possesses vibrissae which engender a tactual orientation to exploration.

With respect to the visual cliff, these differences are generally minimized since the apparatus was designed to control tactual, auditory, and olfactory cues, forcing animals to respond solely on the basis of visual cues. However, this may be questioned for the rat: There is evidence that there is a nonvisual source of depth information. The rat characteristically explores with its forepaws and vibrissae and descends forepaws first; hence the rat can use tactual information for depth discrimination. Two interdependent sources of tactual cues are available from the cliff apparatus: the height of the centerboard and the presence of the glass surface itself. Though Walk \& Gibson (1961) and Walk (1965) have cautioned that a short centerboard may provide tactual information for the rat, quantitative data on this methodological matter are lacking.

The present study was designed to examine the role of tactual cues given by these two apparatus variables. Accordingly, the height of the centerboard, and the presence or absence of the glass surface covering the deep and shallow sides, were varied.

\section{Subjects and Apparatus}

The animals were Holtzman strain male albino rats between 70 and 80 days of age. The apparatus was a copy of the visual cliff, Model II, illustrated in Walk \&
Gibson (1961, Fig. 4). To reduce reflections no overhead lighting was used; lighting was indirect, emanating through small windows located 5-1/2 ft from each end of the apparatus. (Several human observers did not detect the presence of the glass surface.). Incandescent lights fastened to the outsides of the apparatus were adjusted to equate the brightness of the deep and shallow sides. Luminance at the centerboard was approximately $1 \mathrm{ft}-\mathrm{L}$ (a reading of 10 on a Gossen Lunasix light meter). The height of the apparatus, measured from the shallow surface to the floor, was $13 \mathrm{in.}$ The shallow and deep surfaces were covered with a textured material composed of a checkerboard pattern of $3 / 4$ in. red and white squares. The two centerboards were $3 \mathrm{in}$. and 4-1/2 in. in height; both were 3-1/2 in. in width. In two conditions, the glass surface was removed.

Procedure

There were four conditions of testing. The standard cliff with the glass extending over the deep and shallow surface was used with the 3 in. and 4-1/2 in. centerboards (Conditions 1 and 2); the glass was removed from both sides creating a physical cliff and this was used with the two centerboards (Conditions 3 and 4). Each $S$ was placed on the centerboard and observed until it descended or for a maximum of $3 \mathrm{~min}$. Observation by the experimenter was from a position in a line with the centerboard so as not to bias place of descent.

Results

Table 1 summarizes the behavior of the animals in the four conditions. Rats descended only to the shallow side when there was no glass surface extending over the deep and shallow side. Differences in choice of descent for the 3 in. centerboard vs the $4-1 / 2$ in. centerboard resulted only with the glass (Conditions 1 and 2); namely, more deep choices occurred with the shorter centerboard. Finally, more "no descents" were observed with the 4-1/2 In. centerboard (Condition 2) than with the 3 in. centerboard (Condition 1) when a glass surface was used $\left(x^{2}=3.13, p<.01\right.$, based on sum of deep and shallow vs no descent category for the $3 \mathrm{in}$, and $4-1 / 2$ in centerboards). Discussion

The present study has indicated that under some conditions the adult rat will use tactual information for depth discrimination and that the degree of its use varies with its availability. The most direct way to insure that a choice is based on visual information is to use a centerboard high enough to prevent the animal 
Table 1. Performance on the Visual Cliff

Number Descending:

\begin{tabular}{|c|c|c|c|c|c|}
\hline Condition & $\mathrm{N}$ & Shallow & Deep & No Descent & $z$ (shallow vs. deep) \\
\hline \multicolumn{6}{|l|}{ Glass over Deep and Shallow } \\
\hline 1. 3 in. Centerboard & 22 & 12 & 7 & 3 & .92 \\
\hline 2. $4 \frac{1}{2}$ in. Centerboard & 27 & 13 & 3 & 11 & $2.25^{*}$ \\
\hline \multicolumn{6}{|c|}{ No Glass over Deep and Shallow } \\
\hline 3.3 in. Centerboard & 21 & 20 & 0 & 1 & ** \\
\hline 4. $4^{1 / 2}$ in. Centerboard & 21 & 20 & 0 & 1 & ** \\
\hline
\end{tabular}

from touching the shallow-deep plane. The finding that animals with the glass and a $4-1 / 2$ in. centerboard chose the shallow side more often than the deep indicates that the visual cliff is an effective instrument for testing visual depth perception.

The large "no descent" category of Condition 2 reflects the behavior of animals in a situation where tactual cues are reduced by the relatively high elevation of the centerboard. Moreover, it is a situation in which visual and tactual cues give competing information; the tactual cue indicates no difference between the two sides while visually one side involves a fall. The finding in Condition 1 that more animals descended to the deep side with a 3 in. centerboard than with the $4-1 / 2$ in. (again a situation where visual and tactual cues are in opposition) suggests that tactual cues may predominate over visual cues for the adult rat. These results also suggest an explanation of why rats ever perform the maladaptive response of descending to the deep side, a response noted in most studies using the visual cliff apparatus. In the present study this only occurred with the use of the glass, suggesting that tactual cues, at least partially, mediate this response. When tactual cues offer information of a physical cliff (no glass, Conditions 3 and 4) rather than an apparent cliff, no animals descended to the deep side. In general, findings indicate that caution should be exercised in controlling for tactual cues on the cliff. Clearly the species, age, and size of animals is an important consideration for this methodological variable.

\section{References}

WALK, R. D. The study of visual depth and distance perception in animals. In D. S. Lehrman, R. A. Hinde \& E. Shaw (Eds.), Advances in the Study of Behavior. Vol. 1. New York: Academic Press, 1965. WALK, R. D., \& GIBSON, E. J. A comparative and analytical study of visual depth perception. Psychol Monogr., 1961, 75 (15, whole No. 519).

\section{Notes}

1. Supported in part by Public Health Service Grant MH-12878-01 from the National Institute of Mental Health to H. R. Schiffman. The authors acknowledge the assistance given by the Squibb Institute of Medical Research throughout all phases of the study.

2. The principles of laboratory animal care as promulgated by the National Society for Medical Research were observed. 